\title{
Integration von automatisch generierten und manuell konstruierten Prozessmodellen als Grundlage für den Aufbau einer Prozessarchitektur
}

\author{
Susanne Leist, Wolfgang Lichtenegger \\ Lehrstubl für Wirtschaftsinformatik III, insb. Business Engineering, \\ Universität Regensburg
}

\section{Einleitung}

Die systematische Entwicklung und Modellierung einer Unternehmensarchitektur ist nicht nur in der wissenschaftlichen Literatur ein intensiv diskutiertes Themengebiet (Buhl und Heinrich 2004, Zachman 1987), sondern auch ein wichtiges Aufgabengebiet in der Unternehmenspraxis (Jung 2005, Uhl 2004). Die Unternehmensarchitektur soll vor allem die Veränderungen im Unternehmen unterstützen, indem sie die wichtigsten Gestaltungsgegenstände sowie deren gegenseitige Abhängigkeiten im Unternehmen in Form von Modellen beschreibt. Typische Anwendungsgebiete sind u. a. Abdeckungsanalysen, beispielsweise um Lücken oder Redundanzen in der IT-Unterstützung der Geschäftsprozesse zu identifizieren (Aier et al. 2008, S. 294). Auch werden mit Unternehmensarchitekturen Standards definiert, beispielsweise als einheitliche Vorgabe von Prozessabläufen oder ITFunktionalitäten, um historisch gewachsenen, sehr heterogenen Landschaften entgegen zu wirken (Buhl und Heinrich 2004, S. 311). Ein Kernbestandteil der Unternehmensarchitektur ist die Prozessarchitektur (Leist und Zellner 2008, S. 12). Die Bedeutung der Prozessarchitektur zeigt sich nicht nur daran, dass sie in vielen Unternehmensarchitektur Frameworks präsent ist, sondern auch in der Praxis werden die Geschäftsprozesse als wichtigstes Gestaltungsobjekt im Rahmen der Unternehmensarchitektur angesehen (Aier et al. 2008, S. 297).

Nicht nur aus diesem Grunde wird die Modellierung der Prozesse schon seit langer Zeit intensiv diskutiert und es wurden zahlreiche Konzepte und Methoden entwickelt, die hierbei unterstützen. Trotzdem charakterisieren Unternehmen die gegenwärtige Reife der Geschäftsprozesse zur Gestaltung der Unternehmensarchitektur als unzureichend (Aier et al. 2008, S. 297). Dies zeigt sich oft darin, dass nicht mehr aktuelle oder nur unvollständige Prozessmodelle vorliegen. Einen wichtigen Beitrag zur Aktualität und Korrektheit der Modelle kann das Process Mining (van der Aalst et al. 2003), i. S. der automatischen Generierung von Prozessmodel- 
len, leisten. Das Process Mining stützt sich dabei auf Ereignisse, die von den Anwendungssystemen des Unternehmens erzeugt werden. Jedoch ist es häufig nicht möglich, den Prozess auf dieser Basis durchgängig, d. h. von seiner Initiierung bis zu seinem Abschluss, zu generieren. Für den Aufbau einer Prozessarchitektur wird jedoch die durchgängige Erfassung der Prozesse gefordert (Winter 2004, S. 318), was schließlich eine Integration von automatisch generierten und manuell konstruierten Prozessmodellen nahe legt.

Das Ziel des Beitrags ist es, Herausforderungen der Integration von manuell konstruierten und automatisch generierten Prozessmodellen zu untersuchen sowie Ansätze zur Lösung zu geben. Hierzu werden im nachfolgenden zweiten Abschnitt die beiden Modellierungsansätze (manuelle Konstruktion und automatische Generierung) vorgestellt und die Vorteilhaftigkeit der Integration sowie ihr Beitrag im Rahmen der Unternehmensarchitektur begründet. Im dritten Abschnitt werden Herausforderungen bei der Integration identifiziert und deren Grundlagen in einem Morphologischen Kasten zusammengestellt. Anschließend wird in Abschnitt vier die bestehende Literatur nach vorhandenen Lösungsansätzen untersucht. Da keiner der bestehenden Ansätze den Integrationsprozess vollständig unterstützt wird in Abschnitt fünf der eigene Ansatz skizziert. Die Schlussbetrachtung in Abschnitt sechs fasst die wichtigsten Ergebnisse zusammen und stellt den weiteren Forschungsbedarf vor.

\section{Thematische Grundlagen}

\subsection{Prozessarchitektur als Bestandteil der Unternehmensarchitektur}

Für Unternehmensarchitekturen sind Prozesse wichtige Gestaltungsobjekte. Sie werden in der Regel einer eigenständigen Prozess- oder Organisationsebene zugeordnet und gemeinsam mit Organisationseinheiten, Rollen und Informationsobjekten modelliert (Leist und Zellner 2008, S. 13). Der Prozess selbst besteht aus Funktionen (oder Aufgaben) und deren Beziehungen, die zur Entwicklung, Erstellung und dem Vertrieb der Leistungen an den Kunden ausgeführt werden (Österle 1995, S. 19). Aufgrund vieler möglicher Sichten auf die Prozesse eines Unternehmens (z. B. Struktur oder Verhalten) werden die verschiedenen Prozessmodelle im Rahmen einer Prozessarchitektur organisiert, die neben anderen speziellen Architekturen (z. B. Geschäftsarchitektur, IT-Architektur) Bestandteil der Unternehmensarchitektur ist (Winter 2004, S. 318).

Die Prozessmodelle übernehmen eine wichtige Transferfunktion, da mit ihnen die Anforderungen aus der Unternehmensstrategie für die betrieblichen Abläufe umgesetzt werden. Zudem ermöglichen sie, Restriktionen und Potenziale der ITInfrastruktur für die Unternehmensstrategie zu verdeutlichen (vgl. Frank 1994, S. 230-231, Österle 1995, S. 20-21). Darüber hinaus können sie natürlich für viele weitere Zwecke, wie beispielsweise für das Qualitätsmanagement, bei der Einfüh- 
rung von Standardsoftware, dem Compliance-Management oder bei SourcingEntscheidungen, und nicht zuletzt für die Prozessoptimierung verwendet werden (Aier et al. 2008, S. 299).

Für viele der genannten Einsatzzwecke werden Modelle im Ist-Zustand verwendet. Auch in der Praxis werden Ist-Modelle als fester Bestandteil der Unternehmensarchitektur angesehen. Schließlich wird erst durch die Kenntnis des IstZustandes die nötige Transparenz geschaffen, um die Unternehmensarchitektur weiterentwickeln zu können (Aier et al. 2008, S. 299). Eine wichtige Voraussetzung ist dabei, dass die Prozessmodelle aktuell sind und korrekt die Abläufe des Unternehmens darstellen. Die mit dieser Voraussetzung verbundenen Probleme werden im folgenden Abschnitt betrachtet.

\subsection{Modellierung der Prozesse}

Zur Unterstützung der manuellen Konstruktion von Prozessmodellen werden seit den 90er Jahren Ansätze in der Literatur vermehrt publiziert (siehe Ferstl und Sinz 1995; Keller et al. 1992). Neben diesen Ansätzen wurden auch viele Modellierungssprachen entwickelt, die ebenfalls bei der Konstruktion helfen sollen. Trotz dieser zahlreichen Bemühungen besteht nach wie vor eine wesentliche Problemstellung darin, ein gleiches Verständnis über das Modell zwischen Modellierer, Fachexperten und Modellnutzer herzustellen (Leist-Galanos 2006, S. 83). Schwierigkeiten ergeben sich insbesondere daraus, dass die Fachexperten und Modellnutzer gewohnt sind, in natürlicher Sprache zu kommunizieren, während die Modellierer künstliche, konstruierte Sprachen verwenden. Da die Modellnutzer die Anforderungen an die Prozessmodelle bestimmen, die Fachexperten das Basiswissen über die Prozesse zur Verfügung stellen und das Wissen über die Modellierung von den Modellierern beigesteuert wird, ist eine intensive Kommunikation zwischen diesen Gruppen erforderlich. Neben den damit verbundenen hohen Aufwand (Schwegmann und Laske 2005, S. 155), wird als weitere Problemstellung die möglicherweise idealisierte Sicht auf die Prozesse durch die Fachexperten gesehen (van der Aalst 2007, S. 18). Die Nachteile der manuellen Modellierung werden deshalb im hohen Aufwand sowie in möglicherweise nicht korrekten Modellen gesehen.

Eine wichtige Aufgabe des Process Minings ist die automatische Modellgenerierung auf Basis von in der Vergangenheit ausgeführten Prozessinstanzen (van der Aalst und Weijters 2004, S. 232). Grundlage sind auf Ereignissen (z. B. „eine Aufgabe ist auszuführen“, „eine Nachricht wurde ausgetauscht") aufbauende Eventlogs, die sich jeweils auf eine Aktivität (z. B. „Aufgabe ausführen“, „Nachricht austauschen“) beziehen und für jede Prozessinstanz (z. B. „der Kreditantrag von Herrn Schmid wurde geprüft") aufgezeichnet werden (van der Aalst et al. 2005, S. 48). Die Ereignisse können je nach den Anforderungen des gewählten Process Mining-Algorithmus sowohl Vor-als auch Nachereignis der referenzierten Aktivität sein. Aus den erfassten Eventlogs wird anschließend mit Hilfe eines Process Mining-Algorithms automatisch ein Modell abgeleitet, das wiedergibt, wie der Pro- 
zess von den Modellnutzern und Anwendungssystemen tatsächlich ausgeführt wurde (van der Aalst 2005, S. 199). Das Process Mining kann somit die genannten Nachteile der manuellen Konstruktion von Prozessmodellen beheben, d.h. der Erstellungs- bzw. Aktualisierungsaufwand wird reduziert und gleichzeitig werden Modelle erzeugt, die den tatsächlichen Ablauf wiedergeben. Allerdings setzt das Process Mining voraus, dass mit den Anwendungssystemen Eventlogs erfasst werden, die den Prozess durchgängig beschreiben können. Zudem müssen die beobachteten Instanzen repräsentativ sein und es muss eine ausreichend große Anzahl möglicher Durchläufe aufgezeichnet sein (van der Aalst 2005, S. 201). Weitere Herausforderungen ergeben sich beispielsweise bei der Generierung von im Eventlog verborgenen Aktivitäten sowie von Oder-Konstrukten mit vorgegebener Entscheidung. Ebenso ist der Umgang mit verrauschten Daten (fehlende oder fehlerhafte Werte) problematisch. (van der Aalst und Weijters 2004, S. 237-242) Lassen sich diese Herausforderungen nicht beheben, kann das Process Mining keine korrekten Modelle erzeugen.

Die Kombination beider Modellierungstechniken kann in mehrfacher Hinsicht bei der Modellierung von Nutzen sein. So können bspw. über den Vergleich von einem mit Process Mining generierten Modell mit einem manuell konstruierten Modell wichtige Erkenntnisse über gedachte und tatsächliche Ausführung der Aufgaben durch die Modellnutzer und Anwendungssysteme gewonnen werden (van der Aalst 2007, S. 16-17). Darüber hinaus und im Folgenden betrachtet, können beide Modellierungstechniken sich ergänzend zum Aufbau einer Prozessarchitektur eingesetzt werden. Dies ist naheliegend, da Process Mining Nachteile der manuellen Konstruktion beheben kann. Und umgekehrt die manuelle Konstruktion auch dort angewendet werden kann, wenn das Process Mining keine korrekten Modelle generieren kann. Offen ist dabei jedoch, wie die manuell konstruierten mit den automatisch generierten Modellen integriert werden können. Dies wird im Folgenden untersucht.

\section{Herausforderungen bei der Integration der Modelle}

Zielsetzung der Integration ist, aus dem manuell konstruierten und dem automatisch generierten (Ausgangs-)Modellen ein konsistentes (Ziel-)Modell des IstZustandes zu entwickeln. Die Güte dieses Zielmodells ist nicht nur davon abhängig, wie die Ausgangsmodelle integriert werden (z. B. verbunden oder vereinigt), sondern auch von deren Beschaffenheit. Dementsprechend lassen sich die ersten Herausforderungen aus den Eigenschaften der Ausgangsmodelle ableiten.

- Die erste auffälligste Eigenschaft eines Modells ergibt sich aus der verwendeten Modellierungssprache, da die Konstrukte der Modellierungssprache im Wesentlichen die Ausdrucksmächtigkeit des Modells begrenzen. Herausforderungen bei der Integration ergeben sich vor allem, wenn die Ausgangsmodelle in unterschiedlichen Sprachen konstruiert oder generiert werden und deshalb 
die Transformation eines Modells erfordern. Beispielsweise werden Petri Netze häufig als Modellierungssprache von Process Mining Algorithmen verwendet (Tiwari et al. 2008, S. 7), während die EPK bei der manuellen Konstruktion sehr weit verbreitet ist (Peyret et al. 2009, S. 10).

- Bei automatisch generierten Prozessmodellen ist im Weiteren zu unterscheiden mit welchen Algorithmen diese erstellt wurden (Process Mining Algorithmus). Zahlreiche Ansätze zur Generierung von Prozessmodellen werden im ProM Framework (van Dongen et al. 2005; ProM 2009) unterstützt. Bei der oben angesprochenen Herausforderung der Transformation der Modellierungssprachen können sich weitere Probleme ergeben, da manche Algorithmen zur Einhaltung der Sprachsyntax fiktive Ereignisse bzw. Funktionen erstellen.

- Auch die für die Bezeichnung der Modellkonstrukte verwendete Sprache kann unterschiedlich sein. Hier können sich insbesondere Herausforderungen durch auftretende Konflikte (u.a. (Batini et al. 1986, S. 344-347 und Rosemann 1996, S. 187-224)) ergeben. Ein Konflikt zwischen zwei Modellen tritt dann auf, wenn ein gleicher Realitätsausschnitt unterschiedlich abgebildet wird. Dazu gehören beispielsweise Namenskonflikte (Verwendung homonymer oder synonymer Bezeichnungen), Typkonflikte (ein Informationsobjekt wird in den beiden Ausgangsmodellen in unterschiedlichen Typen dargestellt, bspw. als Ereignis oder Funktion) und Strukturkonflikte (gleiche Sachverhalte werden semantisch unterschiedlich modelliert, z. B. durch Verwendung unterschiedlicher Operatoren) (Rosemann 1996, S. 187; 207; 216). Diese Konflikte können durch ein Fachbegriffsmodell (oder Glossar) sowie durch Konventionen, die die Verwendung der Konstrukte festlegen, weitgehend vermieden werden.

- Darüber hinaus ist zu berücksichtigen nach welchem Prinzip die Prozessmodelle entwickelt wurden (Prinzip der Modellierung). So schlagen die Modellierungsmethoden verschiedene Anhaltspunkte vor, an denen Prozesse und Teilprozesse abgegrenzt werden. Zur manuellen Konstruktion wird beispielsweise von Österle (1995, S. 87-88) die Ausrichtung auf Leistungen und Objekte vorgeschlagen, während Ferstl und Sinz (1995, S. 214) Regeln ausgerichtet an den Transaktionen und Objekten vorgeben (Leist-Galanos 2006, S. 289). Dagegen sind automatisch generierte Modelle an die Verfügbarkeit von Eventlogs gebunden und entsprechend nach ihnen ausgerichtet (van der Aalst und Weijters 2004, S. 232). Herausforderungen ergeben sich bei der Integration durch die Angleichung der Modelle, die durch Verwendung unterschiedlicher Modellierungsprinzipien entstanden sind.

- Letztlich kann auch der Detaillierungsgrad, in dem die beiden Ausgangsmodelle vorliegen, unterschiedlich sein. Hieraus können sich Konflikte ergeben und die Herausforderung besteht, eine Angleichung der Modelle vorzunehmen. 
Tabelle 1: Strukturierung der Problemstellung

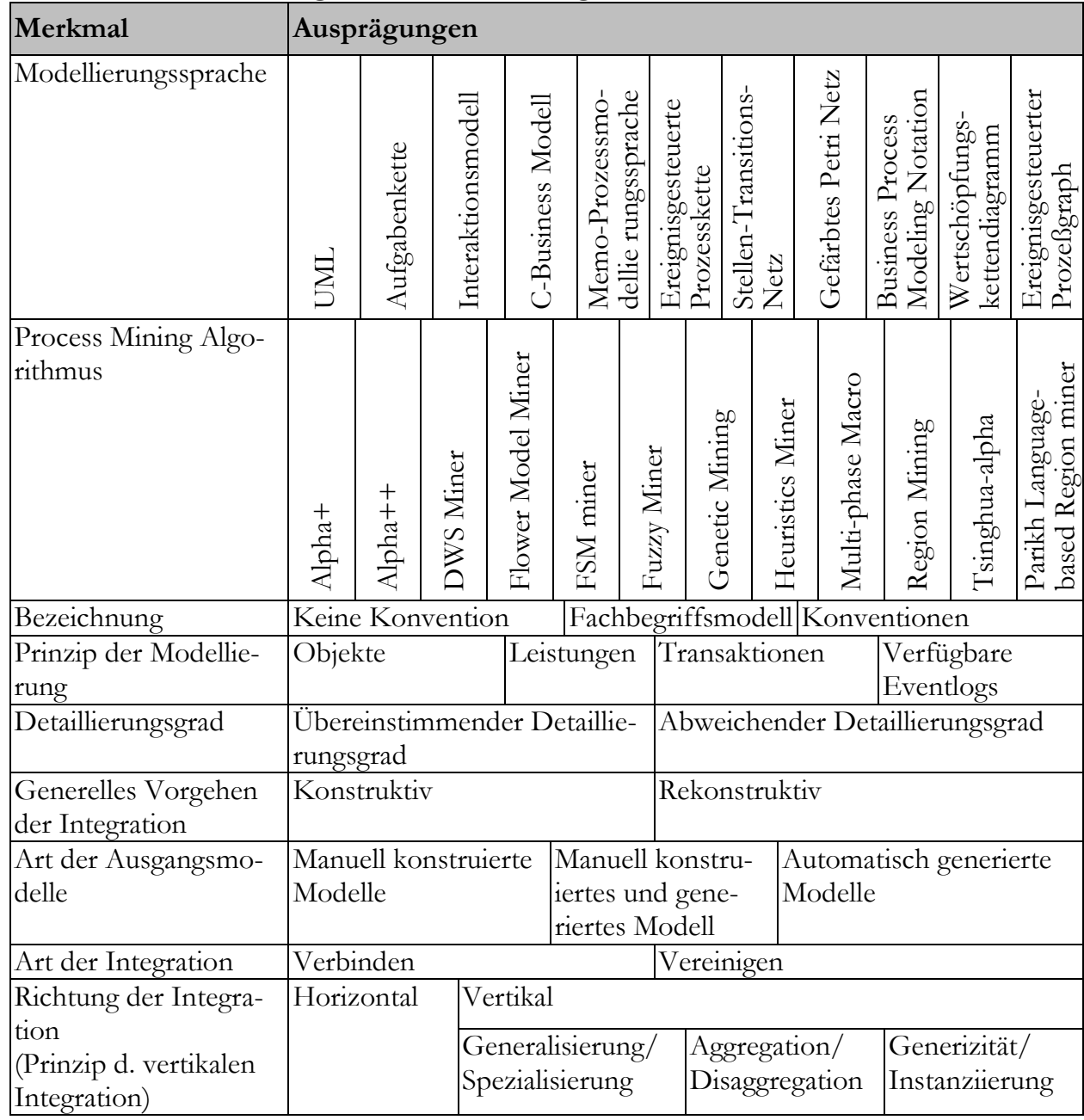

Neben diesen modellbasierten Herausforderungen, die die Voraussetzungen der Integration beeinflussen, sind weitere zu berücksichtigen, die sich durch die Integration selbst ergeben. Im Fokus stehen hierbei Aspekte, die konkret die Ausführung der Integration beeinflussen und bspw. unterschiedliche Aktivitäten zur Integration notwendig machen. Entsprechend ergeben sich unterschiedliche Herausforderungen für den Modellintegrator.

- Ganz maßgeblich wird das Vorgehen der Integration dadurch beeinflusst, ob die Ausgangmodelle bereits generiert bzw. konstruiert sind (generelles Vorgehen der Integration). Liegen bereits generierte und manuell konstruierte Prozessmodelle vor, werden sie rekonstruktiv integriert. Werden die Anforderungen der 
Integration bereits bei der Erstellung der Prozessmodelle einbezogen, so ist das Vorgehen konstruktiv. (Rosemann 1996, S. 172)

- In diesem Beitrag wird die Integration von manuell konstruierten und automatisch generierten Modellen betrachtet (Art der Ausgangsmodelle). Jedoch grundsätzlich denkbar ist auch die Integration von nur automatisch generierten oder nur manuell konstruierten Modellen beim Aufbau einer Prozessarchitektur.

- Auch die Integration selbst kann in unterschiedlicher Art durchgeführt werden (Art der Integration): die Ausgangsmodelle werden verbunden oder vereinigt (Rosemann 1999, S. 5; Jung 2006, S. 38-39).

- Die Ricbtung der Integration kann zwischen horizontal und vertikal differenziert werden (Mertens 2007, S. 5), je nachdem ob die zu integrierenden Ausgangsmodelle auf gleicher Detaillierungsstufe (horizontal) oder auf unterschiedlichen Detaillierungsstufen (vertikal) zu integrieren sind.

- Die vertikale Integration kann unterschiedlichen, grundlegenden Prinzipien folgen (Prinzip zur vertikalen Integration). So werden drei Typen von Beziehungen (vom Brocke 2003, S. 77) zwischen den Ausgangsmodellen auf abstrakter und detaillierter Ebene definiert: Generalisierung/Spezialisierung, Aggregation/Disaggregation, Generizität/Instanziierung.

Sowohl die aufgezählten Modelleigenschaften wie auch die unterschiedlichen Aspekte der Integration werden in einem Morphologischen Kasten (siehe Tabelle 1) zusammengefasst und jeweils um mögliche Ausprägungen ergänzt. Dabei wird nicht der Anspruch erhoben, die Ausprägungen vollständig zu erfassen, was insbesondere bei den Modellierungssprachen zu sehen ist. Der Morphologischen Kasten gibt eine erste Übersicht und strukturiert in einem ersten Ansatz die Vielfalt an Herausforderungen, die bei der Integration zu berücksichtigen sind. Gleichzeitig dient er als Grundlage, Integrationszenarios abzugrenzen, die durch unterschiedliche Pfade anhand einer Auswahl von Merkmalsausprägungen definiert werden. Beispielsweise können automatisch generierte und manuell konstruierte Ausgangsmodelle rekonstruktiv integriert werden. Dabei kann es sich zudem um Ausgangsmodelle handeln, die jeweils mit Petri Netzen dargestellt wurden und die verbunden werden sollen, usw. Ein solches Szenario unterscheidet sich im Hinblick auf die damit verbundenen Herausforderungen bzw. möglicherweise auftretenden Konflikte grundsätzlich von einem Szenario indem die Ausgangsmodelle konstruktiv integriert werden sollen. Im letztgenannten Szenario können Konflikte durch vorausschauende Definition von Konventionen vermieden werden.

\section{Verwandte Arbeiten}

In der Literatur finden sich zahlreiche Ansätze, um den genannten Herausforderungen zu begegnen. Die meisten Beiträge stammen dabei aus den Themenbereichen der Prozessmodellintegration und der Prozessmodellierung. Aufsätze im 
Process Mining beschäftigen sich vor allem mit den Algorithmen zur Generierung der Prozessmodelle (Tiwari et al. 2008, S. 11; Cook und Wolf 1998; van der Aalst et al. 2005), jedoch nicht mit der Integration der Modelle. Da kein Ansatz den Integrationsprozess umfassend unterstützt, wird im Folgenden eine kurze Übersicht über Techniken der bestehenden Ansätzen gegeben, die zumindest teilweise Unterstützung bieten.

Als erste Herausforderung wurde die Transformation der Modellierungssprachen der Ausgangsmodelle (Modellierungssprache und Process Mining Algorithmus) aufgeführt. Gefunden werden Ansätze die direkt Modellierungssprachen auf graphischer Ebene transformieren (Johannsen 2007) oder Modellierungssprachen in ausführbare Sprachen übersetzen (Mendling 2008). Hier von besonderem Interesse sind Ansätze, die EPK-Modelle in Petri-Netze (u. u.) überführen (Dehnert 2009; Lohmann et al. 2009).

Die Verwendung unterschiedlicher Bezeichnungen (Bezeichnung) in den Ausgangsmodellen führt zu zahlreichen Konflikten, die insbesondere im Themenbereich der Qualitätssicherung der Modellierung behandelt werden. Eine Vielzahl von Ansätze zeigen hier Möglichkeiten z.B. durch Vorgabe von Konventionen auf, um solche Konflikte zu vermeiden (Schütte 1998, S. 189-194; Rosemann 1996, S. 87-224). Darüber hinaus lassen sich Ansätze im Bereich der Schemaintegration (Batini et al. 1986) finden. Auch dort werden Vorschläge entwickelt, die sich jedoch auf die Integration von Datenmodellen beziehen und deshalb zunächst noch auf die Problemstellung übertragen werden müssen.

Die Angleichung von Modellen, die mit unterschiedlichem Prinsip der Modellierung erstellt wurden, wird in der Literatur nicht behandelt.

Demgegenüber lassen sich leichter Vorschläge für die Angleichung von Modellen mit unterschiedlichem Detaillierungsgrad finden. Die Vorschläge sind zwar mit der Intention entstanden, dem Modellierer bei der Detaillierung seiner Modelle zu unterstützen. Die Zerlegungsregeln von Ferstl/Sinz (2008, S. 203-204) oder die Kriterien zur Ableitung von Aufgaben aus Prozessen von Österle (1995, S. 86-94) lassen sich jedoch auch für die möglicherweise notwendige Abstraktion oder Detaillierung von Prozessmodellen verwenden.

Das Vorgehen der Integration von manuell konstruierten und automatisch generierten Modellen (Art der Ausgangsmodelle) wird in der Literatur bislang noch nicht behandelt. Zu finden sind Ansätze, die konstruktiv und rekonstruktiv manuell konstruierte Prozessmodelle integrieren und dabei auch die Art der Integration festlegen:

- Priemer (1995) entwickelt mit der Normalisierung von Prozessmodellen ein Vorgehen zur rekonstruktiven Integration im Sinne des Vereinigens von unterschiedlich konstruierten EPKs eines gleichen Prozesses. (Priemer 1995, S. 257-259)

- Rosemann (1996) schlägt zwei detaillierte Vorgehen für die rekonstruktive horizontale Integration im Sinne des Verbindens sowie im Sinne des Vereinigens vor. Zusätzlich werden Hinweise für die konstruktive horizontale Integ- 
ration gegeben. (Rosemann 1996, S. 255-267) Der Ansatz ist spezifiziert für manuell konstruierte EPKs und verwendet Prozesswegweiser als Sprachkonstrukt der horizontalen Integration.

- Schwegmann und Laske (2005) beschreiben einen Ansatz für die kollaborative Entwicklung von Prozessmodellen. Als Integrationsart bei der Konsolidierung wird die Integration im Sinne des Verbindens empfohlen. Als Prinzip der vertikalen Integration ist die Aggregation/Disaggregation angegeben (Schwegmann und Laske 2005, S. 170-171).

- Kupsch (2006) schlägt für manuell konstruierte EPKs ein konstruktives Vorgehen zur dezentralen Erstellung von Prozessteilmodellen mit anschließender horizontaler Integration im Sinne des Verbindens vor. (Kupsch 2006, S. 138)

Neben diesen der Problemstellung sehr nahe kommenden Ansätzen können weitere Anhaltspunkte zur Richtung der Integration und insbesondere zur vertikalen Integration (Prinæip der vertikalen Integration) aus folgenden Ansätzen entnommen werden.

- In der PICTURE-Methode werden Prozessbausteine definiert, die zu einzelnen Prozessteilen kombiniert (horizontale Integration) (Becker et al. 2007, S. 267) und zu Prozessregistern zusammengefasst (vertikale Integration) (Becker et al. 2007, S. 271) werden können.

- Im Rahmen des Process Handbooks werden die rekonstruktiv horizontale wie auch die rekonstruktiv vertikale Integration durch die Koordinationstheorie unterstützt. Ebenso wird die Spezialisierung von Prozessen auf Basis der Fragen wer, was, wo, warum, wann und wie vorgeschlagen. (Malone et al. 1999, S. 429-431; S. 435-436)

- Vom Brocke (2003) beschreibt fünf Konstruktionstechniken für die Referenzmodellierung und leitet aus den unterschiedlichen Beziehungen Sprachkonstrukte ab (vom Brocke 2003, S. 269-312).

- Die Process Map von Heinrich et al. (2009) definiert Kriterien zur Ableitung verschiedener Abstraktionsebenen (konstruktive, vertikale Integration im Sinne des Verbindens) auf Basis der Prinzipien Aggregation/Disaggregation und Generalisierung/Spezialisierung (Heinrich et al. 2009, S. 85-86).

Zusammenfassend lässt sich feststellen, dass es in der Literatur eine Vielzahl von Ansätzen gibt, die bei der Bewältigung einzelner Herausforderungen unterstützen können. Zudem finden sich Ansätze, die auch die Integration von Modellen fokussieren. Jedoch deckt kein Ansatz alle genannten Herausforderungen ab und kein Ansatz behandelt die Integration von manuell konstruierten mit automatisch generierten Modellen. Aufgrund der Vielzahl der Ausprägungen, die im Morphologischen Kasten abgebildet sind, erscheint es nicht erstrebenswert einen Ansatz zu entwickeln, der alle Herausforderungen bewältigt. Viel sinnvoller ist hier für relevante, mit dem Morphologischen Kasten abgrenzbare Szenarien je einen adäquaten Ansatz zu entwickeln. Die Auswahl der relevanten Szenarien kann sich an der Bedeutung der Szenarien für die Praxis orientieren. 


\section{$5 \quad$ Fallbeispiel}

Das Fallbeispiel stellt einen Ansatz zur Integration vor, der für ein bestimmtes Szenario entwickelt wurde. Der Ansatz wird an mehreren Beispielen nach der Design Science Forschungsmethode (Takeda et al. 1990, S. 42-43; Hevner et al. 2004, S. 89) erarbeitet. Dazu werden aus den oben genannten Herausforderungen jeweils Anforderungen für ein Integrationsszenario abgeleitet. Es wird ein Ansatz zur Lösung entworfen, der, soweit möglich, bestehende Arbeiten integriert. AnschlieBend wird der Ansatz in der Praxis eingesetzt. Auch wenn die folgenden Ausführungen sich nur auf den Lieferantenwechselprozess bei einem Unternehmen in der Energiewirtschaft beziehen, erfolgt die Anwendung des Ansatzes an weiteren Fallbeispielen, um diesen zu evaluieren.

Das Unternehmen bietet Endkunden u. a. die Unterstützung beim Wechsel zu einem neuen Energielieferanten an. Zum Nachweis der Einhaltung gesetzlicher Vorgaben (Bundesnetzagentur 2006, S. 10) wird regelmäßig eine Ist-Prozessarchitektur manuell konstruiert, die u. a. EPKs enthält. Da auch Eventlogs für einige Prozessteile vorliegen, ergibt sich die Möglichkeit der automatischen Generierung. Durch Integration von generierten Modellen und bestehender EPKs wird ein positiver Effekt auf die Wirtschaftlichkeit der Modellierung erwartet.

Als Process Mining Algorithmus kommt aufgrund verrauschter Daten der Heuristics Miner zum Einsatz. Bei der Bezeichnung der bestehenden manuell konstruierten EPKs wurden vorhandene Konventionen nicht vollständig eingehalten. Die Abgrenzung automatisch generierbarer Prozessteile erfolgt nach verfügbaren Eventlogs. Aufgrund bestehender manuell konstruierter EPKs und extrahierbarer Eventlogs ergibt sich ein abweichender Detaillierungsgrad der Ausgangsmodelle. Es ist eine horizontale Integration von manuell konstruierten und automatisch generierten Prozessteilen mit rekonstruktivem Vorgehen und der Zielsprache EPK anzustreben. Dieses Szenario besitzt aufgrund der Verbreitung der EPK (Peyret et al. 2009, S. 10) eine hohe Praxisrelevanz.

Die folgenden Anforderungen an die Problemlösung lassen sich mittels der oben genannten Herausforderungen ableiten:

- Das zu entwickelnde Integrationsvorgehen muss für die Modellierungssprachen EPK und heuristisches Netz geeignet sein.

- Durch die Verwendung des Heuristics Miner sind die Anforderungen der Transformation eines heuristischen Netzes in eine EPK sowie die Behandlung von fiktiven Start-, Auslöse- und Endereignissen zu berücksichtigen.

- Die Ausgangsmodelle werden auf Basis verfügbarer Eventlogs abgegrenzt.

- Es sind Namens-, Typ- und Strukturkonflikte und Konflikte mit unterschiedlichen Detaillierungsgraden in den Ausgangsmodellen zu bewältigen.

- Die Prozessmodelle liegen vor, so dass von einer rekonstruktiven Integration auszugehen ist. 
- Da gesetzliche Vorgaben keine alternativen Prozessabläufe erlauben, kann die semantische Beziehung der Ausgangsmodelle als zeitlich nachgelagert definiert werden. Die Ausgangsmodelle werden entsprechend verbunden.

- Weitere Anforderungen an die Richtung der Integration (horizontal) und die Art der Ausgangsmodelle (manuell konstruiert und automatisch generiert) ergeben sich offensichtlich aus dem Szenario.

Für dieses Szenario wurde ein erster Ansatz entwickelt, der vier Schritte umfasst (vgl. Abbildung 1). Der Ansatz kann auf Arbeiten von Rosemann (1996, S. 153275) zur Strukturintegration von Prozessmodellen aufbauen. Dort werden jedoch nicht die Anforderungen behandelt, die sich

- aus der Integration von automatisch generierten und manuell konstruierten Ausgangsmodellen,

- aus der Verwendung des Heuristics Miners,

- aus der Transformation eines heuristischen Netzes in eine EPK

- aus Konflikten mit unterschiedlichen Detaillierungsgraden der Ausgangsmodelle ergeben.

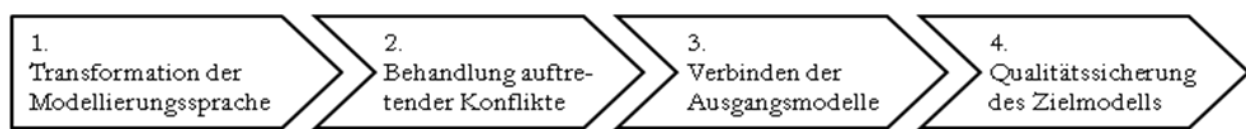

Abbildung 1: Szenariobasiertes Vorgehen der Integration im Fallbeispiel

Als erste Aktivität erfolgt die Transformation des heuristischen Netzes in eine EPK mit anschließender Überprüfung der Transformation. Für die Transformation können die in ProM verfügbaren Conversion Plug-ins (van Dongen et al. 2005, S. 449) verwendet werden, für die Überprüfung der Transformation sind neue Techniken zu entwickeln.

Die zweite Aktivität stellt die Konfliktbehandlung dar. Hierbei sind Namens-, Typ- und Strukturkonflikte sowie Konflikte mit abweichenden Detaillierungsgraden und mit fiktiven Ereignissen aufzulösen. Eine bestimmte Ordnung der Konfliktbehandlung kann nicht festgelegt werden, da sich diese gegenseitig beeinflussen und deshalb von konkreten Ausgangsmodellen abhängig sind. Die Behandlung von Namens-, Typ- und Strukturkonflikten kann von Rosemann (1996, S. 187224) übernommen werden. Weitere Techniken zur Konfliktbehandlung wurden selbst entwickelt. So können abweichende Detaillierungsgrade bspw. dadurch aufgelöst werden, dass die manuell konstruierte EPK auf die Detaillierungsstufe der transformierten EPK gebracht wird. Für die Behandlung von fiktiven Ereignissen in der transformierten EPK wird die Entfernung und anschließende Ergänzung von semantisch sinnvollen Ereignissen vorgeschlagen. Es ist darauf zu achten, dass als Ergebnis der Konfliktbehandlung zwei zeitlich nachgelagerte Prozessteile mit lexikalisch übereinstimmenden End- und Startereignissen entstehen.

Anschließend folgt als dritte Aktivität die Integration im Sinne des Verbindens, welche an Rosemann (1996, S. 257) angelehnt wird. Aufgrund zeitlich nachgelagerten Prozessteile und der lexikalischen Identität von End- und Startereignissen 
können die Teilmodelle durch eine sequentielle Verbindung mittels Prozesswegweiser integriert werden.

Als abschließende Aktivität vier wird das Zielmodell überprüft und ggf. modifiziert, damit beispielsweise festgelegte Konventionen zur Sicherstellung der Qualität des Modells eingehalten werden. Maßnahmen hierzu können von Rosemann (1996, S. 274-275) übernommen werden.

\section{Schlussbetrachtung}

Die systematische Entwicklung und Modellierung der Unternehmensarchitektur wird von der Praxis wie auch der Wissenschaft als ein bedeutendes aber auch umfassendes Aufgabengebiet angesehen. Der vorliegende Beitrag greift die Modellierung der Prozessarchitektur im Ist-Zustand als Themenstellungen heraus und begründet, dass durch Integration von manuell konstruierten und automatisch generierten Modellen die Aktualität, Korrektheit und Vollständigkeit der Architektur erhöht und der Aktualisierungsaufwand reduziert werden kann. Die Schwerpunkte des Beitrags liegen darin, den Integrationsvorgang zu analysieren, Einflussfaktoren zu identifizieren und Ansatzpunkte zur systematischen Durchführung der Integration zu finden. Die identifizierten Einflussfaktoren werden in einem morphologischen Kasten zusammengetragen. Die Untersuchung zeigte, dass die Integration der Modelle aufgrund der vielen Einflussfaktoren ein komplexer Vorgang ist und dass unterschiedliche Integrationsszenarios unterschieden werden müssen. Der morphologische Kasten bildet eine strukturierte Grundlage zur Abgrenzung dieser Szenarios. Die Analyse bestehender Ansätze zeigte, dass keine der untersuchten Methoden die Problemstellung vollständig lösen kann, sich jedoch einzelne Techniken der bestehenden Methoden eigenen, um Teilprobleme zu lösen und damit wiederverwendet werden können. Für ein ausgewähltes Integrationsszenario wurde ein erster Ansatz entwickelt und am Beispiel des Lieferantenwechselprozesses eines Unternehmens in der Energiewirtschaft durchgeführt. Der Ansatz wird aktuell an mehreren Anwendungsfällen validiert. Besondere Herausforderungen ergeben sich dabei insbesondere bei der Transformation der Modellierungssprachen und der Konfliktbehandlung bei unterschiedlichen Bezeichnungen.

Neben der weiteren Validierung des vorgestellten Ansatzes werden zukünftige Arbeiten vor allem in der Abgrenzung der Szenarien sowie der Entwicklung von szenarienbasierten Integrationsmethoden gesehen. Die unterschiedlichen Pfade durch den Morphologischen Kasten können hier als gute Grundlage dienen. Zudem sollte die Frage, für welche Bereiche der Prozessarchitektur eher manuell konstruierte oder eher automatisch generierte Prozessmodelle zu erstellen sind, näher untersucht werden und Auswahlkriterien bereitgestellt werden. 


\section{Literatur}

Aier, S, Riege, C, Winter, R (2008) Unternehmensarchitektur - Literaturüberblick und Stand der Praxis. Wirtschaftsinformatik 50 (4): 292-304.

Batini, C, Lenzerini, M, Navathe, SB (1986) A Comparative Analysis of Methodologies for Database Schema Integration. ACM Computing Surveys Vol. 18, Nr. 4 323-364.

Becker, J, Algermissen, L, Pfeiffer, D, Räckers, M (2007) Bausteinbasierte Modellierung von Prozesslandschaften mit der PICTURE-Methode am Beispiel der Universitätsverwaltung Münster. Wirtschaftsinformatik 49 (4): 267-279.

Buhl, HU, Heinrich, B (2004) Unternehmensarchitekturen in der Praxis Architekturdesign am Reißbrett vs. situationsbedingte Realisierung von Informationssystemen. Wirtschaftsinformatik 46 (4): 311-322.

Bundesnetzagentur (2006) Beschluss BK6-06-009. http://www.bundesnetzagentur.de/media/archive/10893.pdf. Abruf am 22.09.2008.

Cook, JE, Wolf, AL (1998) Discovering models of software processes from eventbased data. ACM Transactions on Software Engineering and Methodology 7 (3): 215-249.

Dehnert, J (2009) Making epcs fit for workflow management. EMISA FORUM 23(1) $12-26$.

Ferstl, OK, Sinz, EJ (1995) Der Ansatz des Semantischen Objektmodells (SOM) zur Modellierung von Geschäftsprozessen. Wirtschaftsinformatik 37 (3): 209220.

Frank, U (1994) Multiperspektivische Unternehmensmodellierung: theoretischer Hintergrund und Entwurf einer objektorientierten Entwicklungsumgebung. Oldenburg, München.

Heinrich, B, Henneberger, M, Leist, S, Zellner, G (2009) The process map as an instrument to standardize processes: design and application at a financial service provider. Information Systems and E-Business Management 7 (1): 81 102.

Hevner, AR, March, ST, Jinsoo, P, Ram, S (2004) Design Science In Information Systems Research. MIS Quarterly 28 (1): 75-105.

Johannsen, F (2007) Transformation von Prozessmodellen: Bewertung XMLbasierter Ansätze. Salzwasser-Verlag, Bremen. 
Jung, E (2005) Das IT-Architekturmodell - Ein Rahmenwerkzeug für die Prozessoptimierung in der HypoVereinsbank. BIT - Banking and Information Technology 6 (2): 62-64.

Jung, R (2006) Architekturen zur Datenintegration - Gestaltungsempfehlungen auf der Basis fachkonzeptueller Anforderungen. Dt. Univ.-Verl., Wiesbaden.

Keller, G, Nüttgens, M, Scheer, A-W (1992) Semantische Prozeßmodellierung auf der Grundlage „Ereignisgesteuerter Prozeßketten (EPK)“. In: Scheer, A-W (Hrsg) Veröffentlichungen des Instituts für Wirtschaftsinformatik - Heft 89, Saarbrücken.

Kupsch, F (2006) Framework zur dezentralen Integration systemübergreifender Geschäftsprozesse. Eul, Lohmar.

Leist-Galanos, S (2006) Methoden zur Unternehmensmodellierung - Vergleich, Anwendungen und Diskussion der Integrationspotentiale. Logos, Berlin.

Leist, S, Zellner, G: Situational Architecture Engineering (SAE) - Improving Strategic Change Through Architecture, International Conference on Information Systems, ICIS 2008 Paris, France, December 14 to 17, 2008.

Lohmann, N, Verbeek, E, Dijkman, R (2009) Petri Net Transformations for Business Processes - A Survey. Transactions on Petri Nets and Other Models of Concurrency II LNCS Volume 5460 46-63.

Malone, TW, Crowston, K, Lee, J, Pentland, B, Dellarocas, C, Wyner, G, Quimby, J, Osborn, CS, Bernstein, A, Herman, G, Klein, M, O’Donnell, E (1999) Tools for Inventing Organizations: Toward a Handbook of Organizational Processes. Management Science 45 (3): 425-443.

Mendling, J (2008) Metrics for Process Models: Empirical Foundations of Verification, Error Prediction, and Guidelines for Correctness. Springer,

Mertens, P (2007) Integrierte Informationsverarbeitung 1 - Operative Systeme in der Industrie 16., überarbeitete Auflage, Gabler, Wiesbaden.

Österle, H (1995) Business Engineering - Prozeß- und Systementwicklung. 2., verbesserte Auflage, Springer, Berlin et al.

Peyret, H, Leganza, G, Smillie, K, An, M (2009) The Forrester Wave: Business Process Analysis, EA Tools, And IT Planning, Q1 2009. Forrester Research, Inc., Cambridge.

Priemer, J (1995) Entscheidungen über die Einsetzbarkeit von Software anhand formaler Modelle. Pro Universitate, Sinzheim.

ProM (2009) Mining Plug-ins contained in ProM. http://is.tm.tue.nl/trac/prom/query? component=Management\&milestone $=$ Mining\&order=priority. Abruf am 2009-08-20. 
Rosemann, M (1996) Komplexitätsmanagement in Prozeßmodellen Methodenspezifische Gestaltungsempfehlungen für die Informationsmodellierung. Gabler, Wiesbaden.

Rosemann, M (1999) Gegenstand und Aufgaben des Integrationsmanagements. In: Scheer, A-W et al. (Hrsg) Integrationsmanagement - Arbeitsbericht des Instituts für Wirtschaftsinformatik Nr.65, Münster.

Schütte (1998) Grundsätze ordnungsmässiger Referenzmodellierung: Konstruktion konfigurations- und anpassungsorientierter Modelle. Gabler, Wiesbaden.

Schwegmann, A, Laske, M (2005) Istmodellierung und Istanalyse. In: Becker, J et al. (Hrsg) Prozessmanagement - Ein Leitfaden zur prozessorientierten Organisationsgestaltung, 5. Auflage, Springer, Berlin et al.

Takeda, H, Veerkamp, P, Tomiyama, T, Yoshikawam, H (1990) Modeling Design Processes AI Magazine 11 (4): 37-48.

Tiwari, A, Turner, CJ, Majeed, B (2008) A review of business process mining state-of-the-art and future trends. Business Process Management Journal 14 (1): 5-22.

Uhl, J (2004) "Unternehmensarchitekturen" ist ein Dauerthema - aber die Ziele bzw. Motivation und damit Schwerpunkte ändern sich, vor allem mit wirtschaftlichen Randbedingungen. Wirtschaftsinformatik 46 (4): 317.

van der Aalst, WMP (2005) Business Alignment: Using Process Mining as a Tool for Delta Analysis and Conformance Testing. Requirements Engineering Journal 10 (3): 198-211.

van der Aalst, WMP: Trends in Business Process Analysis - From Verification to Process Mining, in: Cardoso, J et al. (Hrsg): Proceedings of the 9th International Conference on Enterprise Information Systems (ICEIS 2007), Medeira, Portugal, 2007, 12-22.

van der Aalst, WMP, Alves de Medeiros, AK, Weijters, AJMM: Genetic Process Mining, in: Ciardo, G, Darondeau, P (Hrsg): 26th International Conference on Applications and Theory of Petri Nets (ICATPN 2005), LNCS 3536, 2005, 4869.

van der Aalst, WMP, van Dongen, BF, Herbst, J, Marustera, L, Schimm, G, Weijters, AJMM (2003) Workflow mining: a survey of issues and approaches. Data \& Knowledge Engineering 47 (2): 237-267.

van der Aalst, WMP, Weijters, AJMM (2004) Process Mining: A Research Agenda. Computers in Industry 53 (3): 231-244. 
van Dongen, BF, Alves de Medeiros, AK, Verbeek, HMW, Weijters, AJMM, van der Aalst, WMP (2005) The ProM framework: a new era in process mining tool support. In: Ciardo, G, Darondeau, P (Hrsg) 26th International Conference on Applications and Theory of Petri Nets (ICATPN 2005), LNCS 3536, Springer Heidelberg.

vom Brocke, J (2003) Referenzmodellierung. Gestaltung und Verteilung von Konstruktionsprozessen. Logos, Berlin.

Winter, R (2004) Architektur braucht Management. Wirtschaftsinformatik 46 (4): 317-319.

Zachman, JA (1987) A framework for information systems architecture. IBM Systems Journal Vol. 26, No. 3 276-295. 